\title{
Correction to: Abusive language detection from social media comments using conventional machine learning and deep learning approaches
}

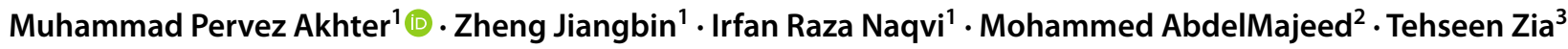

Published online: 22 June 2021

(c) The Author(s), under exclusive licence to Springer-Verlag GmbH Germany, part of Springer Nature 2021

\section{Correction to: Multimedia Systems \\ https://doi.org/10.1007/s00530-021-00784-8}

In the article 'Abusive language detection from social media comments using conventional machine learning and deep learning approaches', by Akhter MP et al., the author list is incorrect. The author Tehseen Zia was added to the authorship list after submission which contravenes the editorial policies of the journal. The correct authorship is Muhammad Pervez Akhter, Zheng Jiangbin, Irfan Raza Naqvi and Mohammed AbdelMajeed.

The original article can be found online at https://doi.org/10.1007/ s00530-021-00784-8.

Muhammad Pervez Akhter

pervezbcs@gmail.com

1 School of Software and Microelectronics,

Northwestern Polytechnical University, Xian 710072,

People's Republic of China

2 School of Computer Science and Technology,

Northwestern Polytechnical University, Xian 710072,

People's Republic of China

3 Department of Computer Science, COMSATS University, Islamabad 44000, Pakistan
Publisher's Note Springer Nature remains neutral with regard to jurisdictional claims in published maps and institutional affiliations. 